\title{
BIT ERROR PROBABILITY ANALYSIS FOR TCM IN SLOW FADING CHANNELS
}

\author{
Chinthananda Tellambura, Qiang Wang, \\ Vijay K. Bhargava, Fellow IEEE \\ Department of Electrical \& Computer Engineering, \\ University of Victoria P.O. Box 3055, Victoria B.C., V8W 3P6 \\ tel(604) 721-8691, email: chintha@sirius.uvic.ca
}

\section{ABSTRACT}

Bit error probability evaluation of Trellis-Coded Modulation (TCM) over fading channels is rather complicated, and the analytical results are limited to loose upper bounds leaving the system designer with the only option of simulation. Cavers and Ho [1] have derived an exact analytical expression for pairwise error-event probability. In this paper, using a different approach, we give an alternative method to compute the exact pairwise error event probability of TCM schemes in Rayleigh or Rician fading channels with perfect side information. For example, for a particular TCM scheme, we find the asymptotic difference between the exact and the upper bound of the pairwise error event probability to be $2.68 \mathrm{~dB}$. As it turns out, for Rayleigh fading, the exact pairwise error probability can be calculated in closed-form. For the case of Rician fading, however, a closed-form expression is apparently impossible to obtain. It is observed that an accurate estimate of the bit error probability can be obtained by evaluating a sufficient number of pairwise error-event probabilities. Hence, this approach is a computationally feasible alternative to the simulation.

\section{INTRODUCTION}

Bit error probability evaluation of TCM in fading channels is extremely complicated and, often, one has to resort to time consuming simulation. For theoretical analysis, Divsalar and Simon [2,5] obtained upper bounds for the Rician and Rayleigh fading channel models with the assumptions of infinite interleaving, slow fading and perfect channel state information. For Rayleigh fading, the upper bound

$$
P(\mathbf{x} \rightarrow \hat{\mathbf{x}}) \leq \prod_{k \in \eta} \frac{1}{1+\frac{E_{s}}{4 N_{0}}\left|x_{k}-\hat{x}_{k}\right|^{2}} .
$$

Being in a product form, these upper bounds allow the evaluation of the union bound on the bit error probability via the transfer function bound ap- proach. References $[2,3,4,5]$ give several examples of such evaluations.

To compute the exact pairwise error event probability for Rician fading channel model, our approach is as follows. We note that the Euclidean distance, appropriately weighted by fading amplitudes, between two code words specifies the conditional pairwise error event probability. And the Euclidean distance can be viewed as a weighted sum of Chi-squared independent random variables, each of which having a simple characteristic function. Therefore, it is possible to compute the exact pairwise error event probability by averaging the conditional pairwise error event probability with respect to the pdf of the weighted Euclidean distance. As will be seen, this approach avoids the residue calculation altogether and instead uses partial factor expansion to evaluate the pairwise error probability. Incidentally, the use of partial factor expansion has been proposed, for a somewhat different context, in $[8]$, too.

This paper is organized as follows. Section 2 derives the basic results. Then we look at several applications of the theory in Section 3. Conclusions are provided in Section 4.

\section{ANALYSIS}

We shall make the same assumptions as in $[1,2]$. Infinite interleaving depth is assumed so as to break the correlation between fading variables leading to the memoryless channel model. Coherent detection is also assumed

As given in [2], the amplitude of the received signal is modeled by the well known Rayleigh distribution

$$
p(\rho)=\left\{\begin{array}{l}
2 \rho \exp \left(-\rho^{2}\right) \rho \geq 0 \\
0 ; \rho<0
\end{array} .\right.
$$

For the sake of brevity, we only consider the Rayleigh model while for Rician model most of our results can be extended, except the closed-form solution. The 
demodulated symbol for the $k$-th symbol period can be denoted by

$$
y_{k}=\rho_{k} e^{j \phi_{k}} x_{k}+n_{k}
$$

where $x_{k}$ denotes the transmitted MPSK symbol at time $k, y_{k}$ denotes the received symbol, $\rho_{k}$ is the normalized random amplitude with the pdf given by Eq. (2), $e^{j \phi_{k}}$ is the phase error term, and $n_{k}$ is a complex-Gaussian noise sample with variance $\frac{N_{0}}{2 \bar{E}}$.

Let $\mathbf{x}$ be the transmitted sequence and the sequence $\hat{\mathbf{x}} \neq \mathbf{x}$ be the one chosen by the Viterbi decoder. Also let $\mathrm{p}(\mathbf{x})$ be the a priori probability of transmitting $\mathbf{x}$ and $a(\mathbf{x}, \hat{\mathbf{x}})$ be the number bit errors associated with this error event. The union upper bound on the average bit error probability is given by

$$
P_{b} \leq \sum_{\mathbf{x}, \hat{\mathbf{x}}} \sum_{\in C} a(\mathbf{x}, \hat{\mathbf{x}}) p(\mathbf{x}) P(\mathbf{x} \rightarrow \hat{\mathbf{x}}) .
$$

Hence, to determine the average bit error rate, the pairwise error event probability should be evaluated first. The pairwise error event probability, $P(\mathbf{x} \rightarrow \hat{\mathbf{x}})$ in Eq. (4), is defined to be the probability of choosing the coded sequence $\hat{\mathbf{x}}=\left(\hat{x}_{1}, \hat{x}_{2}, \ldots, \hat{x}_{N}\right)$ when in reality $x=\left(x_{1}, x_{2}, \ldots, x_{N}\right)$ was transmitted. Thus, by definition, the pairwise error event probability, $P(\mathbf{x} \rightarrow \hat{\mathbf{x}})$ in Eq. $(4)$ is

$$
P(\mathbf{x} \rightarrow \hat{\mathbf{x}})=\operatorname{Pr}\{m(\mathbf{y}, \hat{\mathbf{x}} ; \mathbf{z}) \geq m(\mathbf{y}, \mathbf{x} ; \mathbf{z}) \mid \mathbf{x}\}
$$

where $m(\mathbf{y}, \mathbf{x} ; \mathbf{z})$ is the decoding metric used by the Viterbi decoder, which, due to the facts that additive noise is Gaussian and the availability of perfect channel state information [2,3], takes the form $\left|y_{k}-z_{k} x_{k}\right|^{2}$ where $z_{k}$ is the channel state information. When perfect channel information is available, which we assume, the channel state variable $z_{k}$ becomes $\rho_{k} e^{j \phi_{k}}$.

For the $k$-th step, the maximum-likelihood decoding metric becomes

$$
m\left(y_{k}, x_{k} ; z_{k}\right)=-\left|y_{k}-\rho_{k} e^{j \phi_{k}} x_{k}\right|^{2} .
$$

Combining Eqs. (4), (5), it can be shown that the conditional pairwise error probability is

$$
P(\mathbf{x} \rightarrow \hat{\mathbf{x}} \mid \rho)=Q\left(\sqrt{\frac{\overline{E_{s}}}{2 N_{0}} \sum_{k \in \eta}\left|x_{k}-\hat{x}_{k}\right|^{2} \rho_{k}^{2}}\right)
$$

where $\eta=\left\{i: x_{i} \neq \hat{x}_{i}\right\}$. So $\eta$ is the set of indexes of components, of the two sequences, that differ. And the number of elements of $\eta$ denoted by $L_{\eta}$, or the Hamming distance between the two sequences, is known as the length of the error event.

To remove the conditionality of $P(\mathbf{x} \rightarrow \hat{\mathbf{x}} \mid \rho)$ on $\rho$, the above equation has to be averaged over the joint distribution of fading variables $\rho_{1}, \rho_{2}, \ldots, \rho_{k}$. In order to do that, we derive the characteristic function of the variable, say $\gamma$, defined as

$$
\gamma=\sum_{k \in \eta}\left|x_{k}-\hat{x}_{k}\right|^{2} \rho_{k}^{2}
$$

Since $\gamma$ is a weighted sum of squares of independent random variables, it can be shown that the characteristic function of it is

$$
\phi_{\gamma}(s)=\prod_{k \in \eta} \frac{1}{1+s\left|\mathfrak{x}_{k}-\hat{x}_{k}\right|^{2}}
$$

For the case of Rician fading, the characteristic function of $\gamma$ can be obtained by means of an identity of the modified zero order Bessel function [p.126-9].

Hence, in principle, what has to be done to find the exact pairwise error event is clear. Invert $\phi_{\gamma}(s)$ to obtain $P_{\gamma}(\gamma)$ and then carry out the following expectation.

$$
P(\mathbf{x} \rightarrow \hat{\mathbf{x}})=\int_{0}^{\infty} Q\left(\sqrt{\frac{\bar{E}_{s}}{2 N_{0}} \gamma}\right) P_{\gamma}(\gamma) d \gamma
$$

However, we hasten to add that the inversion is not always necessary and show how to evaluate the expectation directly in Section 3.2.

\subsection{Upper Bounds}

Before evaluating the exact pairwise error bound for some TCM schemes, let us look at the upperbounds of Eq. (10). Using the well known identity, $Q(x) \leq$ $\frac{1}{2} \exp \frac{-x^{2}}{2}$

$$
P(\mathbf{x} \rightarrow \hat{\mathbf{x}}) \leq \frac{1}{2} \int_{0}^{\infty} \exp \left(-\frac{\bar{E}_{s}}{4 N_{0}} \gamma\right) P_{\gamma}(\gamma) d \gamma
$$

Right hand side of the above is clearly equal to $\phi_{\gamma}(s)$ evaluated at $s=\frac{\bar{E}_{s}}{4 N_{0}}$. Hence for Raleigh fad- 
ing, the upper bound is

$$
P(\mathbf{x} \rightarrow \hat{\mathbf{x}}) \leq \frac{1}{2} \prod_{k \in \eta} \frac{1}{1+\frac{\overline{E_{s}}}{4 N_{0}}\left|x_{k}-\hat{x}_{k}\right|^{2}}
$$

which is identical to the upperbounds obtained by Divsalar and Simon except a factor of half.

\subsection{Direct Evaluation}

It is possible to avoid integration twice in order to find pairwise error probability. The Laplace inversion of $\phi_{\gamma}(s)$ given by

$$
P_{\gamma}(\gamma)=\frac{1}{2 \pi j} \int_{\sigma-j \infty}^{\sigma+j \infty} \phi_{\gamma}(s) \exp (s \gamma) d s
$$

Substituting this in Eq. (10), changing the order of integration, and using the standard $\operatorname{erfc}(x)$ averaging over exponential density integral (see $[3,8]$ ), it follows that

$$
P(\mathbf{x} \rightarrow \hat{\mathbf{x}})=\frac{1}{2 \pi j} \int_{\sigma-j \infty}^{\sigma+j \infty} \mathbf{\Psi}(s) d s
$$

where

$$
\Psi(s)=\frac{1}{2}\left\{-1+\sqrt{\frac{\frac{\bar{E}_{s}}{4 N_{0}}}{\frac{\bar{E}_{1}}{4 N_{0}}-s}}\right\} \frac{\phi_{\gamma}(s)}{s} .
$$

Clearly,

$$
P(\mathbf{x} \rightarrow \hat{\mathbf{x}})=\operatorname{Inv} \text { Laplace }\{\Psi(s)\} \text { at } \gamma=0
$$

Furthermore, it is seen that $\Psi(s)$ can always, Rayleigh fading only, be partial factored to terms of form $\frac{1}{s+a}$ and $\frac{1}{(s+a) \sqrt{(b-s)}}$ which have the following two properties:

$$
\frac{1}{2 \pi j} \int_{\sigma-j \infty}^{\sigma+j \infty} \frac{1}{s+a} d s=1
$$

and

$$
\frac{1}{2 \pi j} \int_{\sigma-j \infty}^{\sigma+j \infty} \frac{1}{(s+a) \sqrt{(c-s)}} d s=\frac{1}{\sqrt{(c+a)}}
$$

Hence we need to factor $\phi(s) / s$ into partial fractions and use the above two identities. Also, for error events with repeated Euclidean distances, $1 /(s+a)^{n}$ (where $n \geq 2$ ) terms appear in the above integrand which can simply be handled by differentiating both sides of the above w.r.t $a$. Thus, the total integral can be evaluated by inspection.

\subsection{Incomplete Channel State Infor- mation}

If perfect channel information is not available, the channel side information $z_{k}=e^{j \phi_{k}}$. Note also, if both $\rho_{k}$ and $\phi_{k}$ are unknown, the advantage of coding, of any sort, is lost. In this case the suboptimum decoding metric becomes [5]

$$
m\left(y_{k}, x_{k} ; z_{k}\right)=-\left|y_{k}-e^{j \phi_{k}} x_{k}\right|^{2}
$$

Arguing as we did in section 2 , it can be shown that

$$
P(\mathbf{x} \rightarrow \hat{\mathbf{x}} \mid \rho)=Q\left(\frac{\sum_{k \in \eta}\left|x_{k}-\hat{x}_{k}\right|^{2} \rho_{k}}{\sqrt{\frac{2 N_{0}}{\bar{E}_{*}}} \sum_{k \epsilon \eta}\left|x_{k}-\hat{x}_{k}\right|^{2}}\right)
$$

Notice in this expression we have $\rho$ instead of $\rho^{2}$, which makes a drastic difference as far as the characteristic functions are concerned. So one has to resort to numerical evaluations in this case.

\section{APPLICATIONS}

In this section, we set out to apply the results of section two for several examples. As has been mentioned before, to compute the bit error probability for TCM, a sufficient number of pairwise error event probabilities have to be computed first. A good approximation to the bit error probability is [1]

$$
P_{b} \approx \frac{1}{k} \sum a(\mathbf{x} \rightarrow \hat{\mathbf{x}}) P(\mathrm{x} \rightarrow \hat{\mathbf{x}})
$$

where $a(\mathbf{x} \rightarrow \hat{\mathbf{x}})$ is the number of bit errors associated with the error event and $k$ is the number of input bits per encoding interval.

\subsection{Trellis Coded 4-PSK}

For the TCM shown in Fig 1., consider the pairwise error event between two sequences $\mathbf{x}=\{0,0,0\}$ and 
$\hat{\mathbf{x}}=\{2,1,2\}$. Applying Eqs. (9), (16), (17) and (18), the error event probability given by $(10)$ is

$$
P(\mathbf{x} \rightarrow \hat{\mathbf{x}})=\frac{1}{2}\left(1-\sqrt{\frac{\Gamma}{\Gamma+2}}-\frac{\sqrt{\Gamma}}{(\Gamma+1)^{1.5}}\right)
$$

where $\Gamma=\frac{\overline{E_{s}}}{N_{0}}$.

To compare this exact expression with the bound given in Ineq. 1, it can be shown that for large $\Gamma$ the Taylor expansion of the above equation is roughly $5 / 16 \Gamma^{3}$ while Ineq. 1 gives $2 / \Gamma^{3}$. Therefore, the asymptotic difference between the exact and the upper bound is $2.68 \mathrm{~dB}$. For the second error event, between sequences $\mathbf{x}=\{0,0,0,0\}$ and $\hat{x}=\{2,3,3,2\}$, the error event probability can be obtained similar to the above. Again, it can be shown that the asymptotic difference between the exact and the upper bound is $2.2 \mathrm{~dB}$.

The above two exact error event probabilities are compared with Divsalar and Simon upper bounds in Fig. 2. The difference between the exact and upper bound is just about what has been predicted above. Moreover, it is noticeable that the difference between the first error event (length 3 ) and the second error event (length 4 ) is more than $4 \mathrm{~dB}$, which suggests that only short error events be considered when evaluating the bit error probability. It is also seen that the discrepancy between the exact pairwise error probability and the bound given in [2] decreases as the length of the error event increases.

The overall error upper bound based on transfer function method for this example is given in [3]. Fig. 3. shows the bit error probability obtained by simulation, approximation based on two exact error event probabilities derived in this section and transfer function upper bound given in [3]. One can see that approximate one is indeed close to simulation results, while the upper bound is off by $2-3 \mathrm{~dB}$.

\subsection{Rician Fading}

Here we derive the pairwise error probability for the TCM in Fig. 1 for Rician fading channel. Consider the pairwise error event between two sequences $\mathbf{x}=\{0,0,0\}$ and $\hat{\mathbf{x}}=\{2,1,2\}$. As in Eq. (9), the caracteristic function $\phi_{\gamma}(s)$ can be obtained. Inserting this in Eq. (14) results in an integral which can be computed numerically. Fig. 4 shows the approximate bit error probability, simulation results, and upper bound given in [2]. The approximate bit error probability was obtained using numerical integration of the above equation. It is seen that for $K=5 \mathrm{~dB}$ and $K=10 \mathrm{~dB}$ cases, the approximate bit error probability agrees very closely with the simulation results.

\section{CONCLUSIONS}

In this paper, we have derived an exact expression for the pairwise error event probability, which can be computed rather easily. While our result is valid for both Rayleigh and Rician fading models, for the Rayleigh case the evaluation is particularly simple. For the Rician fading model one has to resort to numerical integration methods. In comparison with Cavers and Ho [1], our method circumvents residue calculation altogether. On the other hand, their method is applicable in more cases, albeit not without considerable computation, while our method is limited to PSK signal sets and ideal channel state information. By computing the exact pairwise error probability for the shortest error event, it is possible to obtain a lower bound for the bit error probability [5]. The close agreement between the approximate bit error probability evaluated using a few short error events and the simulation results suggests that this approximation may alleviate the need for computer simulation.

\section{References}

[1] J. Cavers and P. Ho, " Analysis of the Error Performance of Trellis coded Modulations in Rayleigh Fading Channels," LCCR report, Also IEEE Trans. commun., To appear.

[2] D. Divsalar and M.K. Simon, "The Design of Trellis Coded MPSK for Fading Channels: Performance Criteria", IEEE Trans. on Comm, Vol. 36, No.9, September 1988, pp. 300-308.

[3] E. Biglieri and P.J. McLane, "Uniform Distance and Error Probability Properties of TCM schemes," IEEE Trans. on Comm., Vol. 39, No. 1, Jan 1991.

[4] R.G. Mckay, P.J. McLane, and E. Biglieri," Analytical Performance Bounds on Average Bit Error Probability for Trellis Coded PSK Transmitted Over Fading Channels," ICC 1989.

[5] E. Biglieri, D. Divsalar, P.J. McLane, and M.K. Simon, Introduction to Trellis-Coded Modulation with Applications, Macmilan., 1991.

[6] J.G. Proakis, Digital Communications. New York: McGraw-Hill, 1983.

[7] M. Schwartz, W.R. Bennett, and S. Stein, Communication Systems and Techniques. New York: McGraw-Hill, 1966.

[8] Q. Wang, "Capacity Evaluation of Cellular CDMA" Final report prepared for MPR Teltech Ltd., under contract MPR-H24A260(91104), Nov. 20, 1991.

[9] I.N. Sneddon, Special functions of mathematical physics and chemistry. London: Longman, 1980 


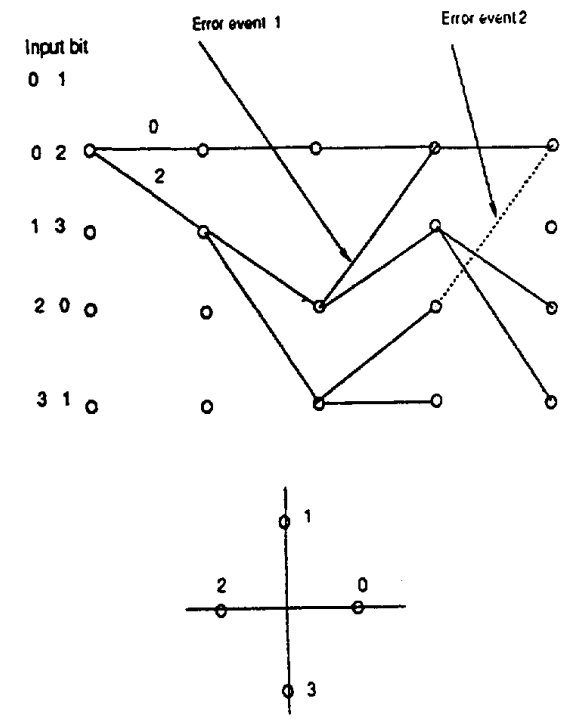

Figure 1: Trellis diagram for 4-slate, APSk TCM Scheme.

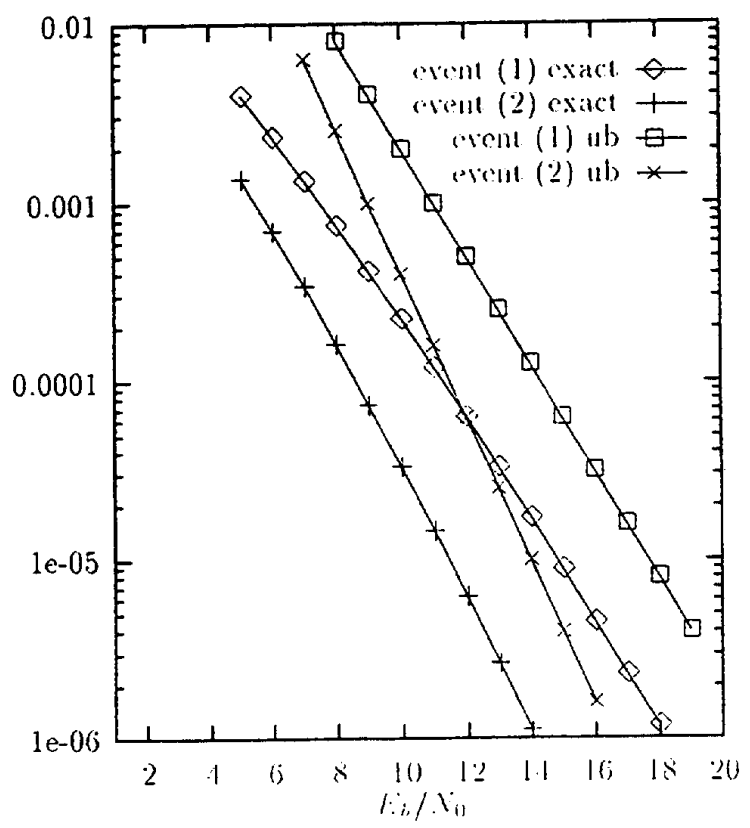

Figure 2: Upper houmds and lixact pairwise errot

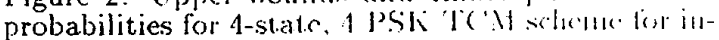
dependent Rayleigh fauling.

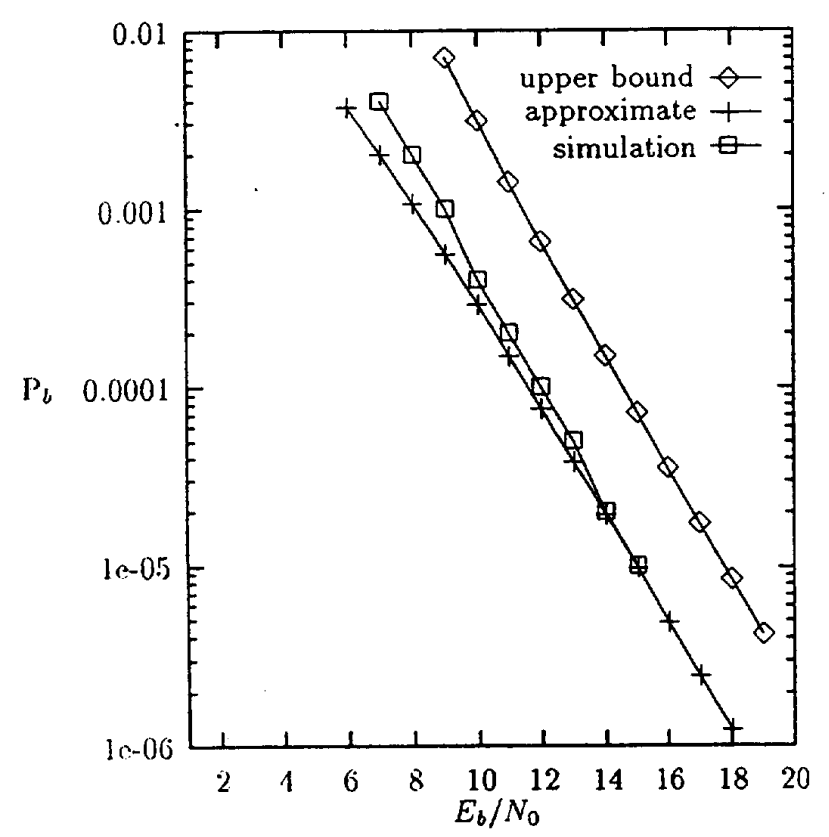

Figure 3: lpper bound, approximate and simulated BLER for 4-l'SK ' $\Gamma$ CM scheme for Rayleigh fading.

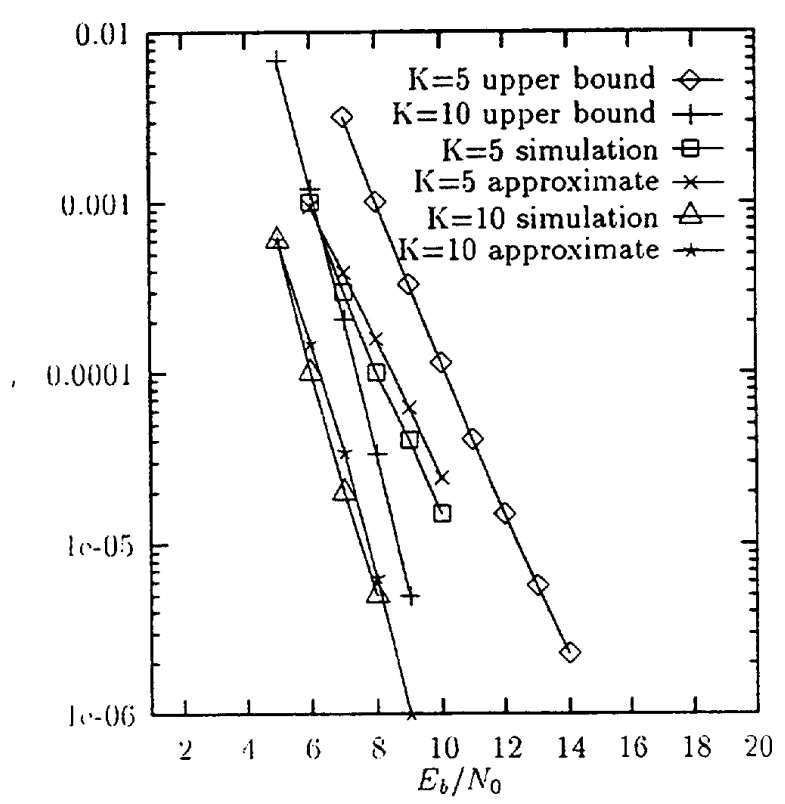

Finure 1: l:pper hound, approximate and simulated BlR lor A-PSK l'M scheme for Rician fading. 